\title{
Combined bioinformatics analysis reveals gene expression and DNA methylation patterns in osteoarthritis
}

\author{
DELEI SONG, WEI QI, MING LV, CHUN YUAN, KANGSONG TIAN and FENG ZHANG \\ Trauma Department of Orthopedics, Zibo Central Hospital, Zibo, Shandong 255036, P.R. China
}

Received April 5, 2017; Accepted February 15, 2018

DOI: $10.3892 / \mathrm{mmr} .2018 .8874$

\begin{abstract}
Osteoarthritis (OA) is a common type of arthritis, which may cause pain and disability. Alterations in gene expression and DNA methylation have been proven to be associated with the development of OA. The aim of the present study was to identify potential therapeutic targets and associated processes for OA via the combined analysis of gene expression and DNA methylation datasets. The gene expression and DNA methylation profiles were obtained from the Gene Expression Omnibus, and differentially expressed genes (DEGs) and differentially methylated sites (DMSs) were identified in the present study, using R programming software. The enriched functions of DEGs and DMSs were obtained via the Database for Annotation, Visualization and Integrated Discovery. Finally, cross analysis of DEGs and DMSs was performed to identify genes that exhibited differential expression and methylation simultaneously. The protein-protein interaction (PPI) network of overlaps between DEGs and DMSs was obtained using the Human Protein Reference Database; the topological properties of PPI network overlaps were additionally obtained. Hub genes in the PPI network were further confirmed via reverse transcription-quantitative polymerase chain reaction (RT-qPCR). The results of the present study revealed that the majority of DEGs and DMSs were upregulated and hypomethylated in patients with OA, respectively. DEGs and DMSs were primarily involved in inflammatory, immune and gene expression regulation-associated processes and pathways. Cross analysis revealed 30 genes that exhibited differential expression and methylation in OA simultaneously. Topological analysis of the PPI network revealed that numerous genes, including $\mathrm{G}$ protein subunit $\alpha 1$ (GNAI1), runt related transcription factor 2 (RUNX2) and integrin subunit $\beta 2$ (ITGB2), may be involved in the development of OA. Additionally, RT-qPCR analysis of GNAI1, RUNX2 and ITGB2 provided further
\end{abstract}

Correspondence to: Professor Feng Zhang, Trauma Department of Orthopedics, Zibo Central Hospital, 54 Gongqingtuan West Road, Zibo, Shandong 255036, P.R. China

E-mail: fengzhangzb@126.com

Key words: bioinformatics, DNA methylation, Gene Expression Omnibus, gene expression, osteoarthritis confirmation. Numerous known and novel therapeutic targets were obtained via network analysis. The results of the present study may be beneficial for the diagnosis and treatment of OA.

\section{Introduction}

Osteoarthritis (OA) is the most common type of arthritis and a leading cause of pain and disability, which places a great burden on the economy of health and reduces quality of life (1-3). OA involves the degeneration of numerous tissues, including subchondral bone, ligaments, muscle, tendons, and the meniscus and synovium (1). Numerous factors may affect OA progression, including age, gender, obesity, genetics and joint injury (4); however, how these factors affect the development of $\mathrm{OA}$ requires further investigation and no effective method has been developed for the relief of pain. In addition, molecular biology studies have identified numerous biomarkers and biological processes that contribute to OA, including the erosion of the extracellular matrix (5), the expression of chemokines (chemokine C-C ligands 9 and 5, and interleukin-8) (6) and the upregulation of inflammatory genes (7). Further investigation into the molecular events associated with cartilage degeneration is required.

Over the past decades, the development of high-throughput technologies has resulted in the large amount of accumulation of omics data for various complex diseases. For OA, gene expression profiling via microarray or high-throughput sequencing has become a promising method for the analysis of the mechanisms underlying its initiation and progression (8). For example, Rasheed et al (9) performed an integrated study of microRNA (miRNA) expression profiles in OA chondrocytes and OA-associated genes, and identified numerous miRNAs associated with the development of OA. Sun et al (10) reported several potential biomarkers for OA via differential expression and network analysis based on gene microarray datasets. Microarray analysis in the study of Loeser et al (11) indicated the link between age-associated differences in gene expression and the development of OA. In addition, epigenetic modifications serve important roles in gene expression regulation, and DNA methylation is one of the most common types of epigenetic modification. Recently, an increasing number of studies have focused on the associations between methylation status and the progression of OA $(12,13)$. In contrast to cancer, in which $\mathrm{CpG}$ sites are frequently hypermethylated, the majority of studies 
investigating OA reported a higher frequency of hypomethylation $(14,15)$. DNA methylation may also affect the allelic imbalance of specific small nucleotide polymorphisms, and thus the development of OA (16). Combined analysis of gene expression and DNA methylation profiles may contribute to the screening of potential biomarkers of OA, early diagnosis and treatment; to the best of our knowledge, an investigation into this is yet to be performed.

In the present study, combined analysis of publicly accessible gene expression and DNA methylation microarray datasets of OA was conducted. Functional enrichment and network analysis was performed for the identification of potential biomarkers. Numerous known and novel targets were obtained and their involvement in OA was further confirmed via reverse transcription-quantitative polymerase chain reaction (RT-qPCR) analysis.

\section{Materials and methods}

Microarray datasets. The publicly accessible data were all obtained from the Gene Expression Omnibus (GEO, www.ncbi.nlm.nih.gov/geo). Gene expression profiles deposited by Klinger et al (17), accession no. GSE43923, containing six samples (three osteophytic cartilage and three corresponding articular cartilage samples from the knee joints of patients with OA) were employed in the present study. The genome-wide expression profiles were quantified using the commercial gene microarray GPL570 [HG-U133_ Plus_2] Affymetrix Human Genome U133 Plus 2.0 Array (Affymetrix; Thermo Fisher Scientific, Inc., Waltham, MA, USA). The DNA methylation profiles (GSE73626) (18) of five hip OA, six knee OA and seven hip healthy cartilage samples were detected via Illumina HumanMethylation450 BeadChip assay (Illumina, Inc., San Diego, CA, USA), which contains $>480,000$ methylation sites, covering 99\% of RefSeq (https://www.ncbi.nlm.nih.gov/refseq/) genes and 96\% of University of California, Santa Cruz (http://genome.ucsc. edu/)-defined $\mathrm{CpG}$ sites with an average of $17 \mathrm{CpG}$ sites/gene across different genomic regions, including the promoter, 5 untranslated region (UTR), first exon, gene body, intergenic and 3'UTR.

Microarray data analysis. The present study conducted differential expression analysis for osteophytic and articular cartilage samples from patients with OA. The raw CEL data were imported into R version 3.2.2 (http://www.R-project. org/) and normalized via the affy package (19); subsequently, the limma package (20) was used for the screening of differentially expressed genes (DEGs) with the criteria of fold change $>1.5$ and false discovery rate $(F D R)<0.05$. For the methylation dataset, site-level analysis was performed based on the Illumina Methylation Analyzer package (Illumina, Inc.) (21) to obtain the differentially methylated CpG sites (DMSs) between hip/knee OA cartilage and healthy cartilage samples, with thresholds of $\mathrm{db}$ value $>0.2$ and FDR $<0.05$. DMSs were mapped to the corresponding genes (DMGs) and genomic regions based on the full annotation file of the microarray and following this, cross analysis was performed via the 'intersect' function of R version 3.2.2 (http://www.R-project.org/) using DEGs and DMGs to reveal overlapping genes. In addition, differences between distributions of DMSs relative to $\mathrm{CpG}$ islands and genes were compared using the $\chi^{2}$ test.

Functional clustering analysis. Investigation into the functions of enriched DEGs and DMGs may improve understanding of their involvement in OA. In the present study, functional clustering analysis of DEGs and DMGs based on the Database for Annotation, Visualization and Integrated Discovery (DAVID; david.abcc.ncifcrf.gov) (22) was conducted. Clusters with an enrichment score $>1$, and Gene Ontology (GO) terms and Kyoto Encyclopedia of Genes and Genomes (KEGG; www. genome.jp/kegg) pathways with $\mathrm{P}<0.05$ were retained in the present study.

Protein-protein interaction network analysis. Genes are likely to function together rather than alone in complex diseases; hub nodes in the network may represent key biomarkers. In the present study, protein-protein interaction (PPI) network analysis was performed to investigate the overlaps between DEGs and DMGs based on the Human Protein Reference Database (HPRD; www.hprd.org) (23). The network was visualized using Cytoscape 3.6.0 software (http://www.cytoscape.org/), and the topological property of every gene [degree (number of direct interactions in the network)] was additionally analyzed for the assessment of their importance.

$R T-q P C R$. Normal and OA tissues were obtained from the articular cartilage of 26 females and 20 males with a median age of 56.35 years (43.58-69.12 years) between April 2012 and April 2015 in Zibo Central Hospital (Zibo, China). Patients exhibiting temporomandibular joint pain that were not suffering from any form of rheumatic disease or cancer were included in the present study. The study was approved by the ethics committee of Zibo Central Hospital. Written informed consent was obtained from all patients.

Total RNA was extracted from OA and normal tissues (50-100 mg) using an RNeasy Mini kit (Qiagen GmbH, Hilden, Germany) and quantified with a NanoDrop system (Thermo Fisher Scientific, Inc.), and subsequently subjected to RT-qPCR using EasyScript Reverse Transcriptase kit (Promega Corporation, Madison, WI, USA). The temperature protocol used for RT was as follows: $95^{\circ} \mathrm{C}$ for $10 \mathrm{~min}, 55^{\circ} \mathrm{C}$ for $1 \mathrm{~min}$ and $68^{\circ} \mathrm{C}$ for $10 \mathrm{~min}$.

The 7500 Real-Time PCR system (Applied Biosystems; Thermo Fisher Scientific, Inc.) was used for qPCR. Reactions were conducted in triplicate in each reaction tube using AceQ qPCR SYBR Green Master Mix (Vazyme Biotech Co., Ltd., Nanjing, China). The temperature protocol used for qPCR was as follows: $94^{\circ} \mathrm{C}$ for $5 \mathrm{~min}$; followed by 40 cycles of $95^{\circ} \mathrm{C}$ for $10 \mathrm{sec}$ and $60^{\circ} \mathrm{C}$ for $30 \mathrm{sec}$; followed by $95^{\circ} \mathrm{C}$ for $15 \mathrm{sec}, 60^{\circ} \mathrm{C}$ for $60 \mathrm{sec}$ and $95^{\circ} \mathrm{C}$ for $15 \mathrm{sec}$. Data were analyzed via the $2^{-\Delta \Delta \mathrm{Cq}}$ method using GAPDH as internal control (24). Primer sequences were: G protein subunit $\alpha 1$ (GNAI1) forward, 5'-TTAGGGCTATGGGGAGGTTGA-3', and reverse, 5'-GGTACTCTCGGGATCTGTTGAAA-3'; runt related transcription factor 2 (RUNX2) forward, 5'-TGG TTACTGTCATGGCGGGTA-3' and reverse, 5'-TCTCAG ATCGTTGAACCTTGCTA-3'; integrin subunit $\beta 2$ (ITGB2) forward, 5'-TGCGTCCTCTCTCAGGAGTG-3' and reverse, 5'-GGTCCATGATGTCGTCAGCC-3'; and GAPDH forward, 
Table I. Previously identified biomarkers of osteoarthritis with the PMID of each corresponding reference.

Author, date

Gene

(Refs.)

Mabey T et al, 2014

Angiopoietin 2

Gao W et al, 2013

Valverde-Franco G, 2016

Ephrin B2

Leijten JCH, 2013

Gremlin 1 DAN family BMP antagonist

Yi J, 2016

Fan Y, 2017

Integrin subunit $\beta 2$

Hopwood B, 2007

Xiao JL, 2015

Runt related transcription factor 2

Liao L, 2017

A

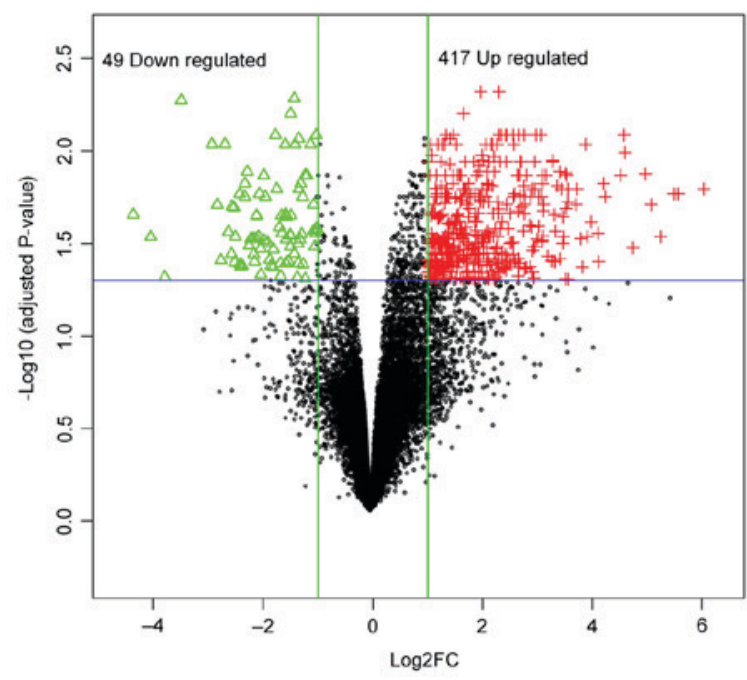

B

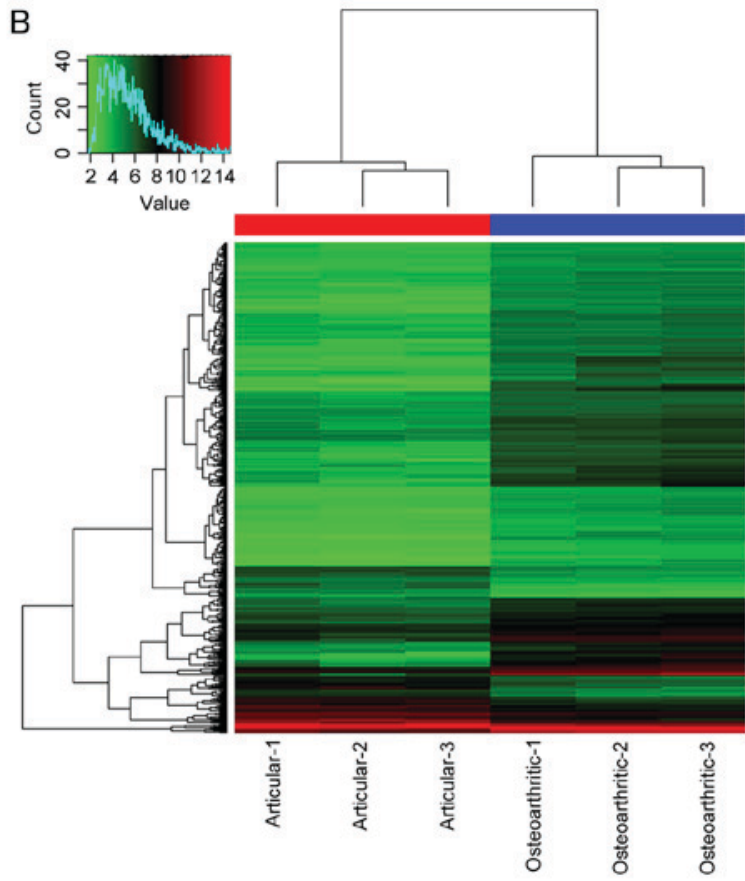

Figure 1. Gene expression profile analysis. (A) Distribution of upregulated and downregulated genes. Red plus signs and green triangles represent upregulated and downregulated genes in patients with osteoarthritis, respectively. Black circles are non-DEGs. (B) Two-way supervised clustering of DEGs and samples, with blue and red coloring indicating low and high expression levels, respectively. DEGs, differentially expressed genes.

5'-ACAACTTTGGTATCGTGGAAGG-3' and reverse, 5'-GCCATCACGCCACAGTTTC-3'.

Statistical analysis. R version 3.2.2 (http://www.R-project. org/) was used for all of the statistical analysis. The relative mRNA expression levels in the RT-qPCR analysis were presented as the mean \pm standard deviation of the three replicates. RT-qPCR data were analyzed using a Student's t-test. $\mathrm{P}<0.05$ was considered to indicate a statistically significant difference.

\section{Results}

Gene expression profile analysis. The raw microarray data was normalized and used for the following differential expression analysis. As a result, a total of 466 genes were detected to be DEGs in osteophytic cartilage samples compared with articular samples, which contained 49 downregulated and 417 upregulated genes (Fig. 1A). The two-way supervised clustering indicated notable differences between osteophytic and articular cartilage samples from patients with OA, with blue and red coloring indicating low and high expression levels, respectively (Fig. 1B). The full list of DEGs is provided in Table I.

DMSs. Comparisons were performed between DMSs in hip/knee cartilage samples and healthy cartilage samples. As presented in Fig. 2A, the $\beta$ values of OA hip compared with healthy hip tissue, and OA knee compared with healthy knee tissue, of all $\mathrm{CpG}$ sites in the microarray were obtained. The $\beta$ values for OA hip compared with healthy hip tissue, 
A

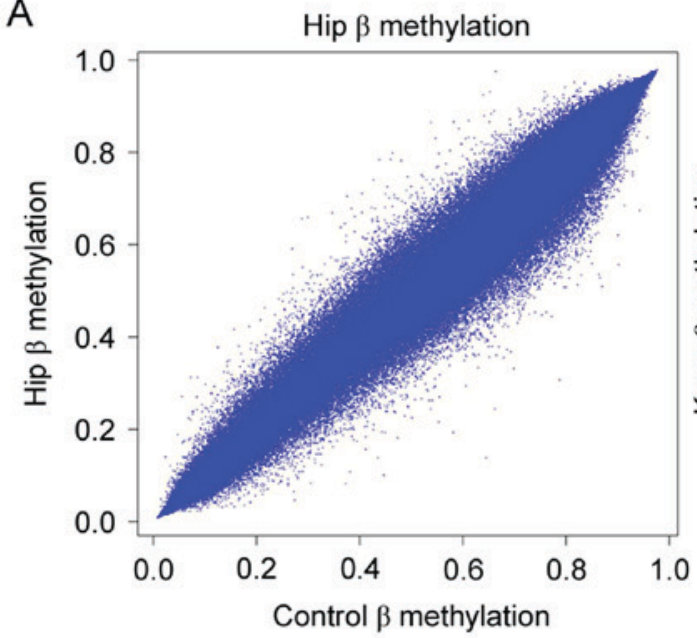

B

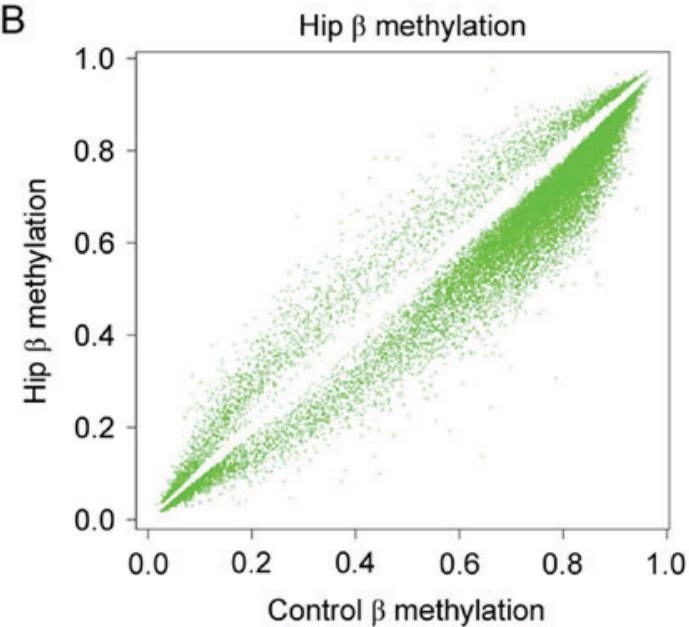

C

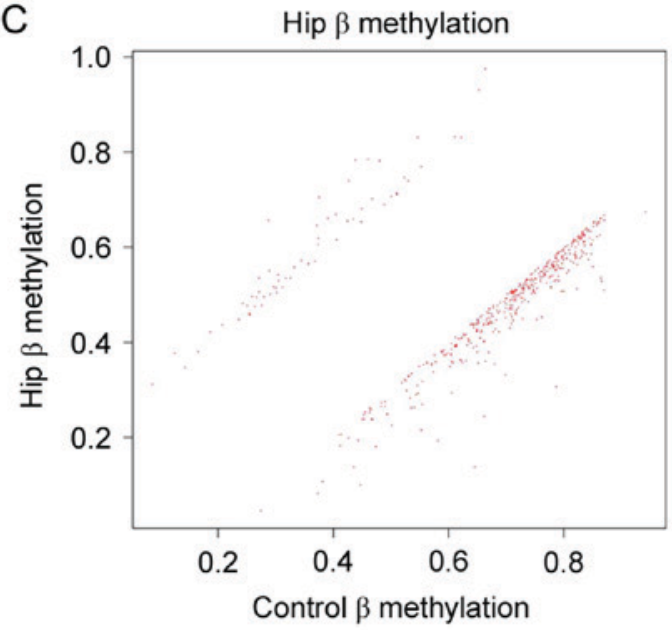

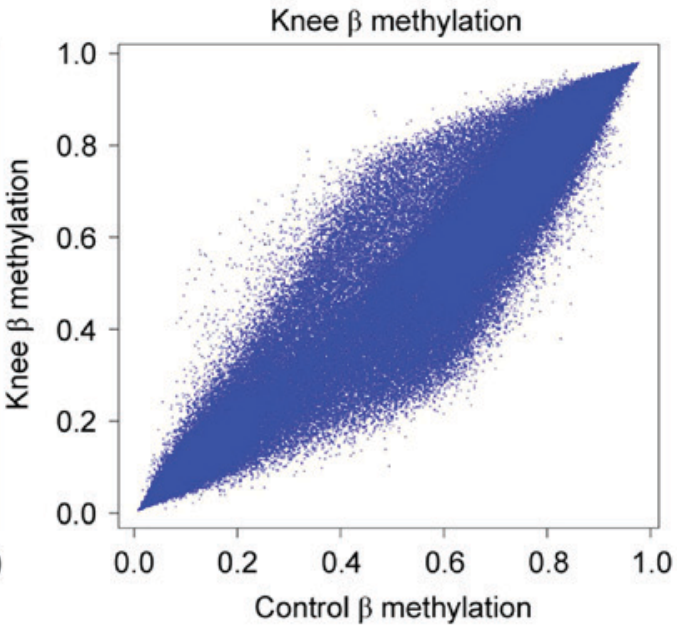
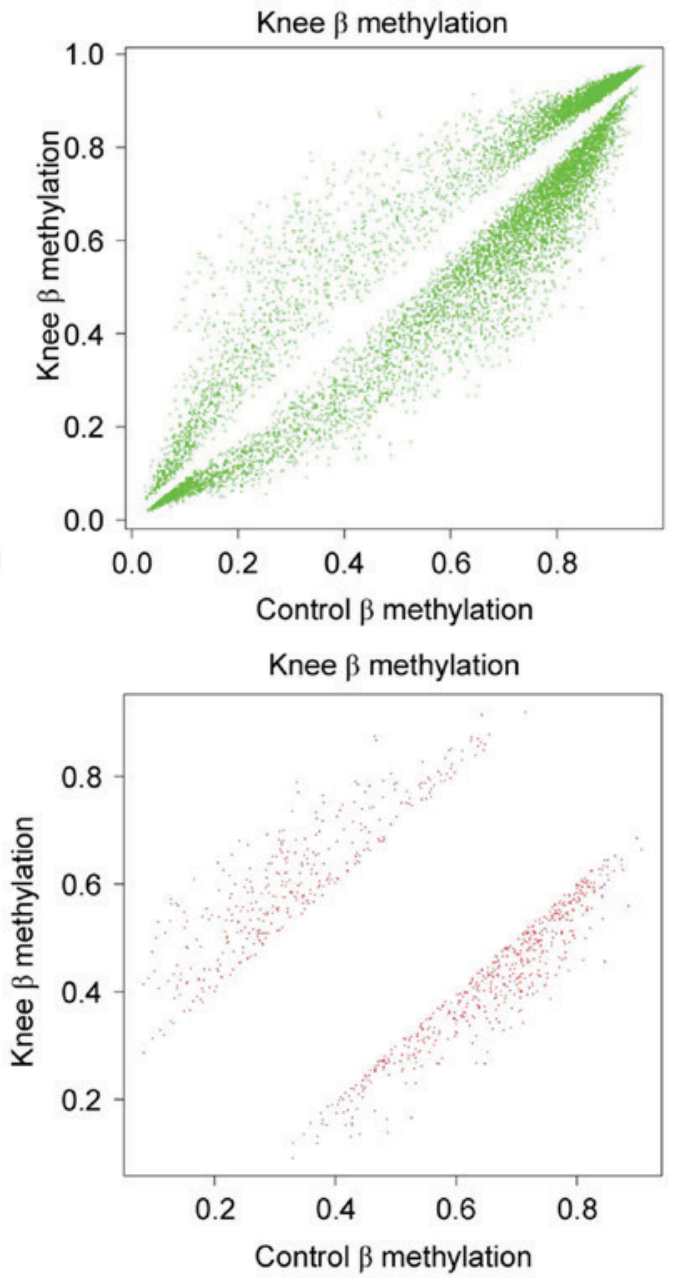

Figure 2. Scatter plot of $\beta$ values in the hip and knee cartilage of patients with OA compared with control samples. (A) $\beta$ values of all the CpG sites in the Illumina HumanMethylation450 BeadChip assay of OA hip cartilage compared with control hip cartilage (left panel), and OA knee cartilage compared with control knee cartilage (right panel). (B) $\beta$ values of $\mathrm{CpG}$ sites satisfying the criterion of $\mathrm{P}<0.05$ of OA hip cartilage compared with control hip cartilage (left panel), and OA knee cartilage compared with control knee cartilage (right panel). (C) $\beta$ values of $\mathrm{CpG}$ sites satisfying the criteria of $\mathrm{P}<0.05$ and $\delta \beta>0.2$ of $\mathrm{OA}$ hip cartilage compared with control hip cartilage (left panel), and OA knee cartilage compared with control knee cartilage (right panel). OA, osteoarthritis.

and OA knee compared with healthy knee tissue, of $\mathrm{CpG}$ sites satisfied the criterion of $\mathrm{P}<0.05$; the frequencies of hypomethylated sites were increased compared with hypermethylated sites in OA hip and knee samples (Fig. 2B). Additionally, the number of hypomethylated sites with $\mathrm{P}<0.05$ and $\mathrm{db}>0.2$ were increased compared with hypermethylated sites (Fig. 2C).
This was consistent with the results of the DEG analysis (the number of upregulated genes was increased compared with downregulated genes), as hypermethylation of the promoter may result in the downregulation of the corresponding gene.

To improve understanding of the functional significance of DMSs, the functional locations of DMSs were investigated. As 
A Functional genomic distribuition
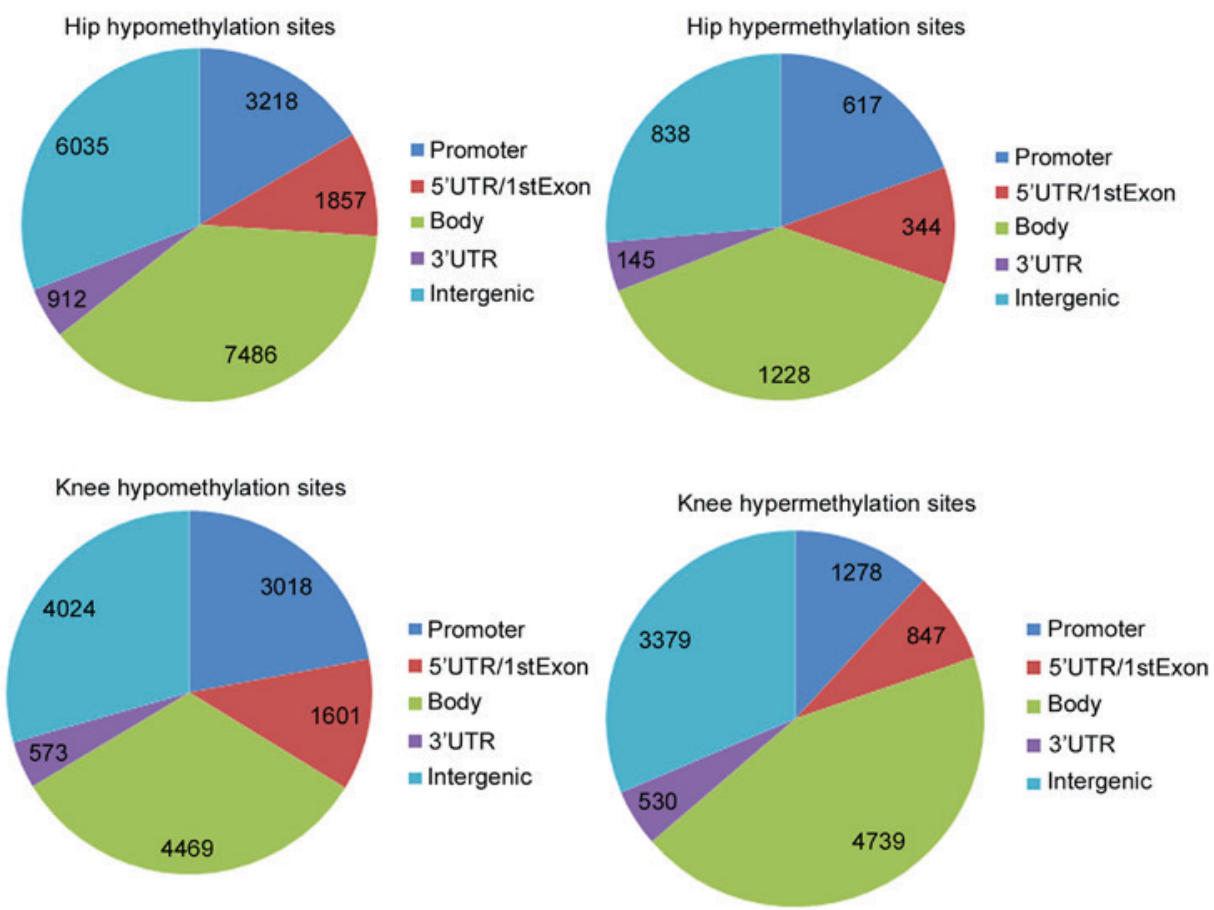

B Neighborhood location
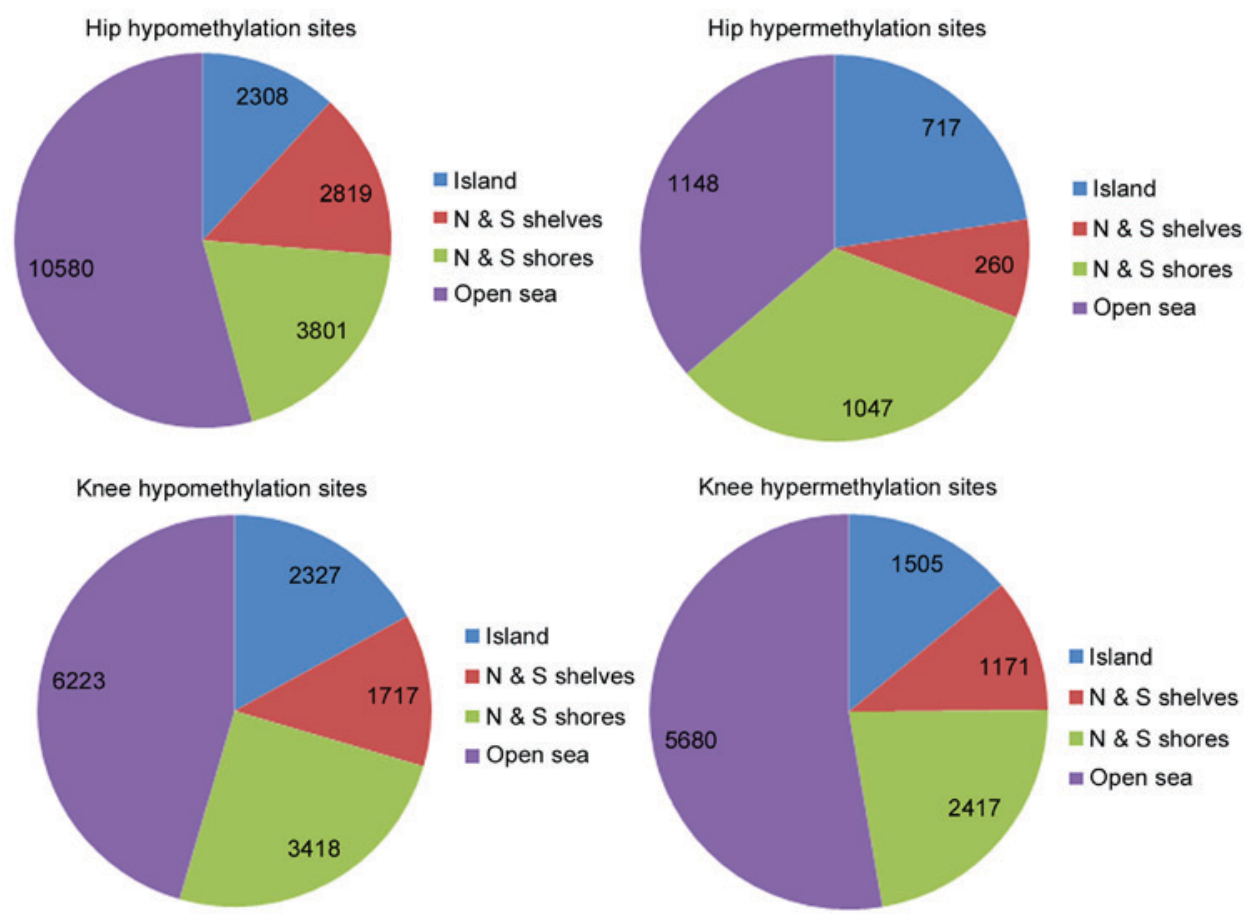

Figure 3. Functional genomic and neighborhood location distributions of differentially methylated sites. (A) Functional genomic distributions of hypomethylation and hypermethylation $\mathrm{CpG}$ sites in $\mathrm{OA}$ hip and knee cartilage. (B) Neighborhood location distributions of hypomethylation and hypermethylation CpG sites in OA hip and knee cartilage. OA, osteoarthritis; UTR, untranslated region.

presented in Fig. 3A, the majority of the DMSs were reported to be in intergenic, gene body and promoter regions. Additionally, four neighborhood locations were defined in the Illumina HumanMethylation450 BeadChip assay: 31\% CpG islands, 23\% shores (0-2 kb from canonical CpG islands), $10 \%$ shelves (2-4 kb from canonical) and open sea (rest of the sequence). Consistent with the BeadChip assay, the majority of the hypo- and hypermethylated sites in hip and knee cartilage samples were detected in open sea, and following the north and south shores (upstream and downstream shores). To investigate the associations between functional and neighborhood locations with differential methylation status, a $\chi^{2}$ test for data presented in Fig. 3. The results indicated $\mathrm{P}<5 \times 10^{-7}$ in all of the cases, demonstrating that functional and neighborhood locations are associated with differential methylation status. One-way clustering of DMSs of hip and knee cartilage samples is presented in Fig. 4. 
A

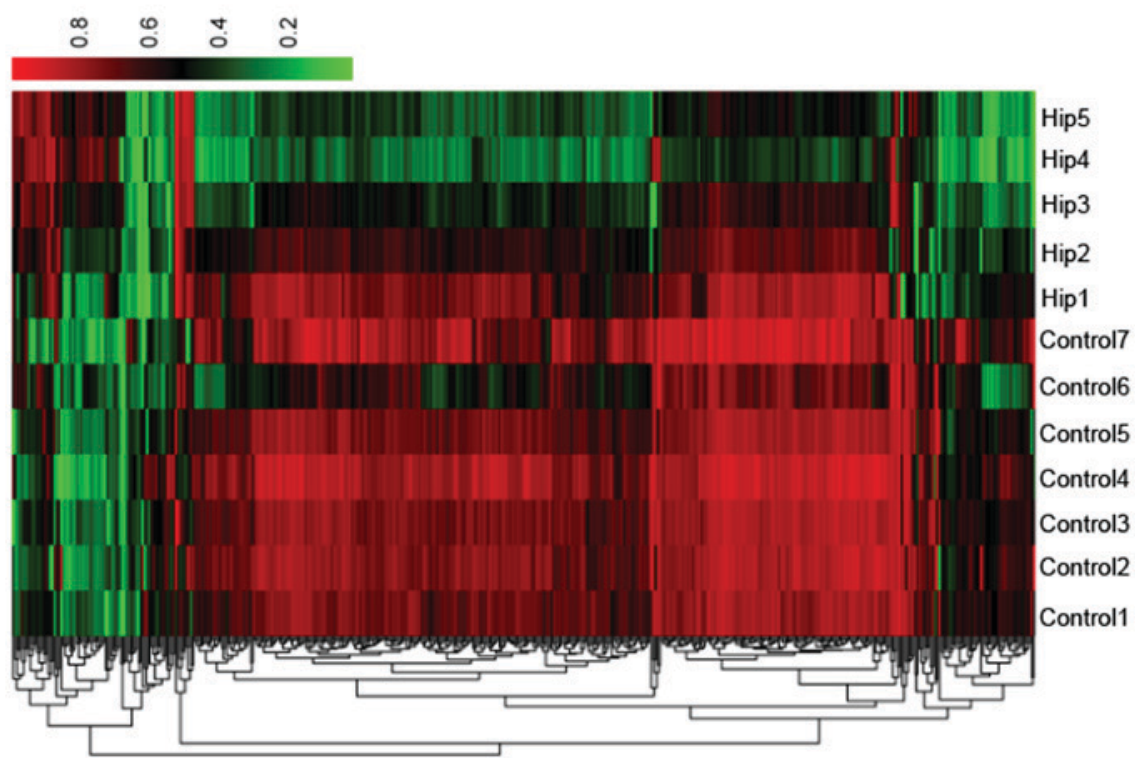

B

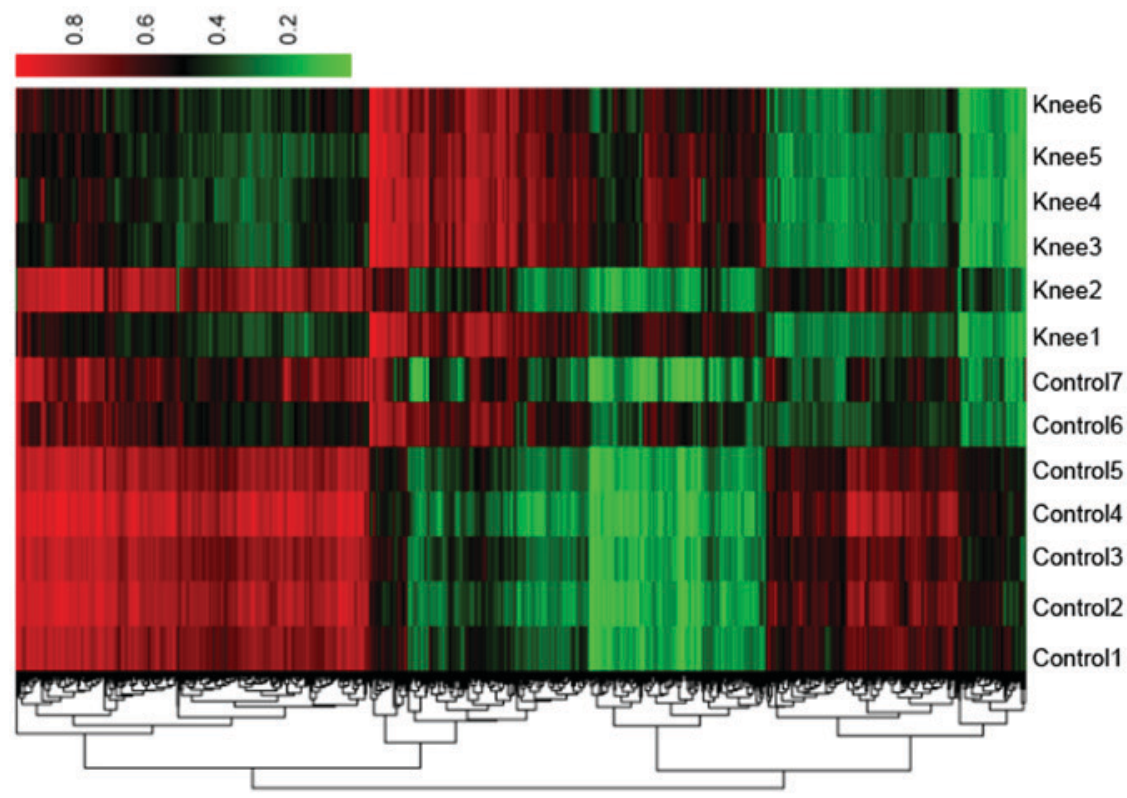

Figure 4. Heatmap of differentially methylated sites in osteoarthritic hip cartilage and knee cartilage samples. (A) Heatmap of DMSs in osteoarthritic hip cartilage. (B) Heatmap of DMSs in knee cartilage samples. DMSs, differentially methylated CpG sites.

Functional clusters. Functional clustering analysis in DAVID resulted in three functional clusters for DEGs and DMGs. DEGs were primarily involved in the GO terms and KEGG pathways that were associated with 'cell structure', 'inflammatory and immune response', 'substance synthetic' and 'metabolic'. 'Guanosine 5'-triphosphaate (GTP)ase activity', 'gene expression regulation' and 'inflammatory and immune response' were reported to be significantly enriched in DMGs (data not shown).

Network analysis. Network topological properties are important representations of their roles in specific biological processes and diseases. In the present study, 30 overlaps were obtained among DMGs of hip and knee cartilage samples and DEGs; 20 of these overlaps were reported to interact with other genes from the HPRD. PPI networks are presented in
Fig. 5. GNAI1 directly interacted with 50 genes, which was markedly higher compared with the degree of the other 19 overlaps in the network, potentially indicating its important roles in the development of OA. Table I includes the five previously identified biomarkers of OA and their corresponding PMID nos.

RT-qPCR analysis. A total of three hub genes, GNAI1, RUNX2 and integrin subunit $\beta 2$ (ITGB2), were subjected to RT-qPCR analysis for the quantification of their relative abundance in OA and control samples. The results of the RT-qPCR analysis were consistent with the results of the gene microarray analysis; the relative mRNA expression levels of GNAI1, RUNX2 and ITGB2 in OA samples were significantly increased compared with the control samples (Fig. 6). 


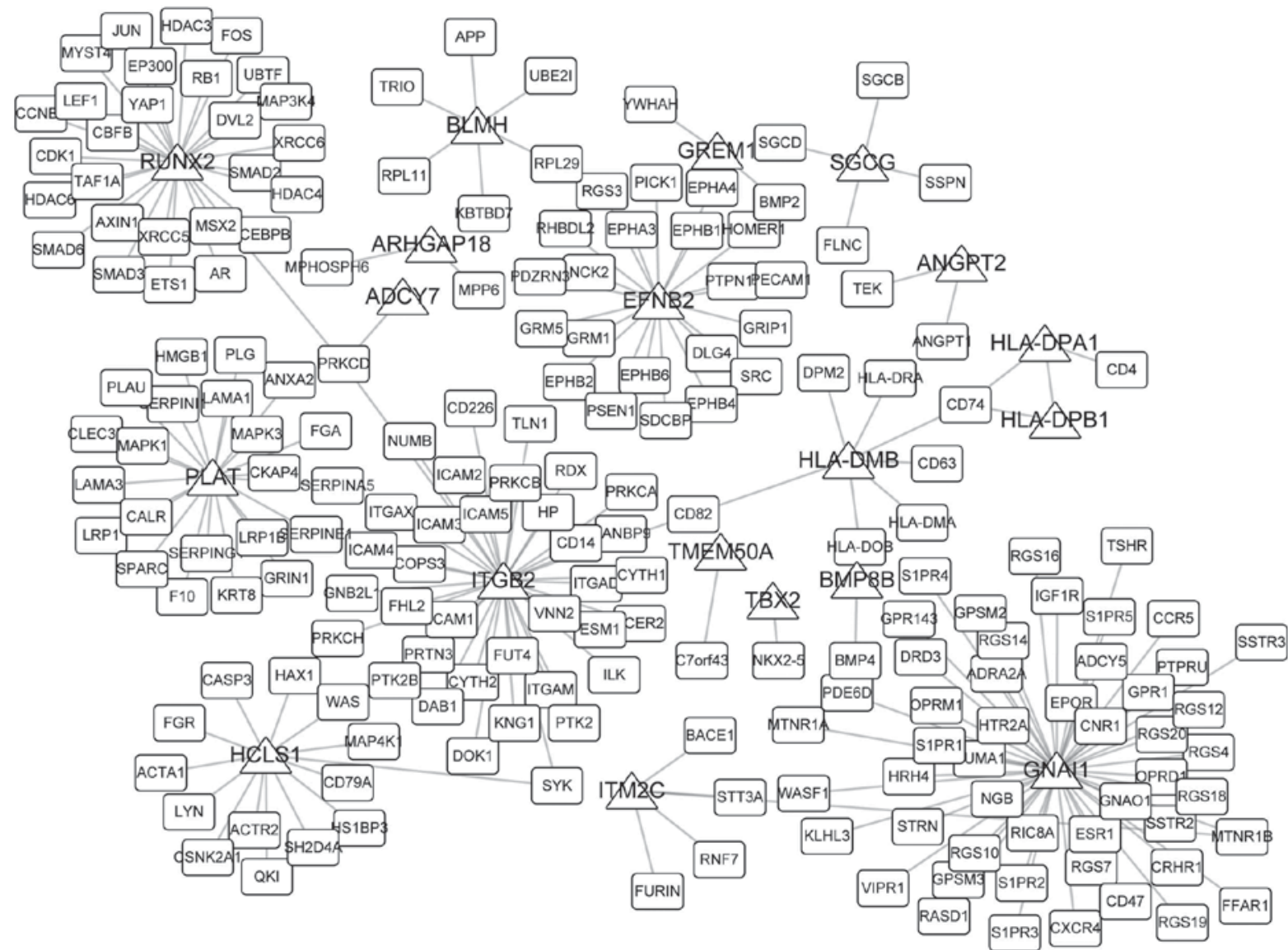

Figure 5. Protein-protein interaction network of the overlaps between differentially expressed genes and differentially methylated CpG sites of corresponding genes. Triangles represent overlapped genes and circles are other genes that interacted with these overlaps.

A
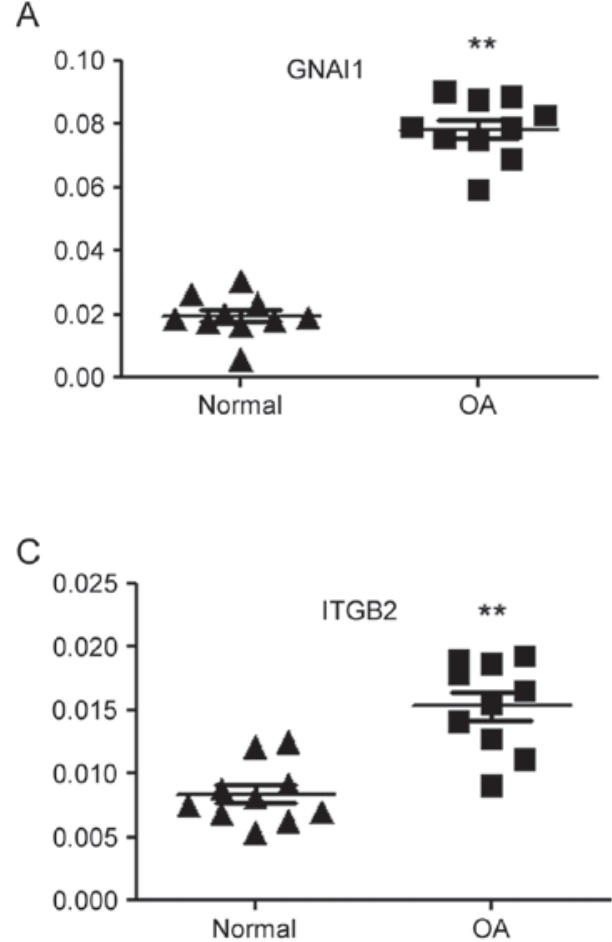

B

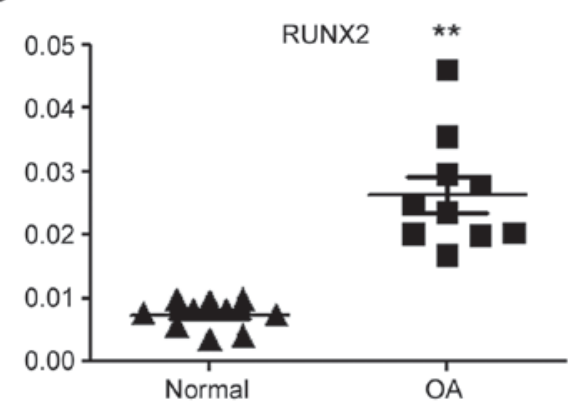

Figure 6. RT-qPCR analyses of GNAI1, RUNX2 and ITGB2. (A) Relative mRNA expression levels of GNAI1 in control and OA samples. (B) Relative mRNA expression levels of RUNX2 in control and OA samples. (C) Relative mRNA expression levels of ITGB2 in control and OA samples. The abundance of each of the three genes was quantified by RT-qPCR, and all were consistent with the gene microarray analysis. ${ }^{* *} \mathrm{P}<0.01$ vs. normal control. GNAI1, G protein subunit $\alpha 1$; ITGB2, integrin subunit $\beta 2$; OA, osteoarthritis; RT-qPCR, reverse transcription-quantitative polymerase chain reaction; RUNX2, runt related transcription factor 2 . 


\section{Discussion}

$\mathrm{OA}$ is the most common degenerative disease of the synovial membrane, comprising the destruction and loss of articular tissues (25). The initiation and development of OA have been reported to be associated with numerous factors, including age, joint injury, obesity and chronic inflammation, in addition to genetic factors, including epigenetic modification and altered gene or miRNA expression (26). In-depth understanding of gene regulation in OA may contribute to diagnosis and treatment. For this purpose, DNA methylation and gene expression analysis of hip and knee cartilage of OA patients was performed, and potential targets for OA were identified and verified in the present study.

DNA methylation has been revealed to repress gene expression by blocking the sites at the promoter where transcription factors bind; hypermethylation of the promoter is associated with no or low transcription (27). In the present study, the majority of the DEGs were observed to be upregulated and DMSs were hypomethylated, consistent with the roles of DNA methylation. Unlike cancer, which is characterized by the hypermethylation of tumor suppressor genes, genome-wide hypomethylation in OA has been widely observed previously (28-30) and in the present study. It is known that OA is influenced by inflammatory chemokines (31), which was also demonstrated by the functional enrichment analysis of the present study. The hypomethylation and increased expression of a number of inflammation-associated genes have been observed to be associated with OA, including IL-8 (32), nuclear factor- $\kappa \mathrm{B}$ (33) and pleckstrin (34), which may serve as therapeutic targets for OA. In the present study, numerous genes that were differentially expressed and methylated simultaneously were identified, including ITGB2, GNAI1 and RUNX2. To the best of our knowledge, the roles of the aforementioned genes in the development of OA have not been investigated; the RT-qPCR analysis conducted in the present study revealed their relative abundance in OA and adjacent tissues, which may indicate their association with the progression of OA. Furthermore, numerous genes were observed to directly interact with other genes in the PPI network, including RUNX2, bleomycin hydrolase and gremlin 1 DAN family BMP antagonist (GREM1). The majority of these genes have been demonstrated to be closely associated with the progression of OA; for example, RUNX2 polymorphisms may affect temporomandibular joint $\mathrm{OA}$ in females (35) and may influence the expression of other genes in OA (36). The mRNA expression levels of GREM1 are correlated with OA and may be regulated by OA-associated factors (37); in addition, GREM1 is a key regulator of synoviocyte hyperplasia and invasiveness (38). Valverde-Franco et al (39) reported that ephrin-B2 may be essential for normal bone growth, and its absence may lead to knee and hip OA. In addition, a number of genes with a high degree in the PPI network have not been proven to be associated with the development of OA. For example, GNAI1, also known as Gi, is a protein that can hydrolyze GTP and interact with other proteins, and T cell differentiation may alter its structure (40). Additionally, the altered expression of GNAI1 was observed to be associated with the progression of inflammatory and immune diseases $(41,42)$, and may be considered to be a novel biomarker for OA.

Certain limitations of the present study were noted. Only three genes were analyzed via RT-qPCR and future studies are required to investigate more genes. Furthermore, osteophytes may be considered to contribute to regeneration in OA joints; however, further studies investigating the differences regarding whole genome expression between OA and healthy tissues should be employed for further analysis in the present study.

In conclusion, the present study provided a pipeline for the combined analysis of gene expression and DNA methylation datasets. In addition, numerous known and potential novel markers were proposed in the present study, which may contribute to diagnosis and treatment targets for OA; however, further investigation is required for confirmation of the functions exhibited by these markers.

\section{Acknowledgements}

Not applicable.

\section{Funding}

No funding was received.

\section{Availability of data and materials}

The datasets analyzed in the present study are available in the GEO repository, www.ncbi.nlm.nih.gov/geo/query/acc. cgi?acc=GSE43923 and www.ncbi.nlm.nih.gov/geo/query/acc. cgi?acc $=$ GSE73626.

\section{Authors' contributions}

DS analyzed and interpreted the microarray datasets and produced the manuscript. WQ and ML modified the syntax; CY and KT designed experiments of the present study. FZ made substantial contributions to the conception and design of the study, and submitted the manuscript. All authors read and approved the final manuscript.

\section{Ethics approval and consent to participate}

The present study was approved by the ethics committee of Zibo Central Hospital, Zibo, China. Written informed consent was obtained from all patients.

\section{Consent for publication}

Not applicable.

\section{Competing interests}

The authors declare that they have no competing interests.

\section{References}

1. Pan Q, O'Connor MI, Coutts RD, Hyzy SL, Olivares-Navarrete R, Schwartz Z and Boyan BD: Characterization of osteoarthritic human knees indicates potential sex differences. Biol Sex Differ 7: 27, 2016. 
2. Young IC, Chuang ST, Hsu CH, Sun YJ, Liu HC, Chen YS and Lin FH: Protective effects of aucubin on osteoarthritic chondrocyte model induced by hydrogen peroxide and mechanical stimulus. BMC Complement Altern Med 17: 91, 2017.

3. Rhee J, Park SH, Kim SK, Kim JH, Ha CW, Chun $\mathrm{CH}$ and Chun JS: Inhibition of BATF/JUN transcriptional activity protects against osteoarthritic cartilage destruction. Ann Rheum Dis 76: 427-434, 2017.

4. Zhang M,Egan B and Wang J: Epigenetic mechanisms underlying the aberrant catabolic and anabolic activities of osteoarthritic chondrocytes. Int J Biochem Cell Biol 67: 101-109, 2015.

5. Schwager J, Hoeller U, Wolfram S and Richard N: Rose hip and its constituent galactolipids confer cartilage protection by modulating cytokine, and chemokine expression. BMC Complement Altern Med 11: 105, 2011.

6. Nair A, Gan J, Bush-Joseph C, Verma N, Tetreault MW, Saha K, Margulis A, Fogg L and Scanzello CR: Synovial chemokine expression and relationship with knee symptoms in patients with meniscal tears. Osteoarthritis Cartilage 23: 1158-1164, 2015.

7. Hashimoto S, Rai MF, Gill CS, Zhang Z, Sandell LJ and Clohisy JC: Molecular characterization of articular cartilage from young adults with femoroacetabular impingement. J Bone Joint Surg Am 95: 1457-1464, 2013.

8. Park R and Ji JD: Unique gene expression profile in osteoarthritis synovium compared with cartilage: Analysis of publicly accessible microarray datasets. Rheumatol Int 36: 819-827, 2016.

9. Rasheed Z, Al-Shobaili HA, Rasheed N, Al Salloom AA, Al-Shaya O, Mahmood A, Alajez NM, Alghamdi AS and Mehana el-SE: Integrated study of globally expressed micrornas in il-1beta-stimulated human osteoarthritis chondrocytes and osteoarthritis relevant genes: A microarray and bioinformatics analysis. Nucleosides Nucleotides Nucleic Acids 35: 335-355, 2016.

10. Sun J, Yan B, Yin W and Zhang X: Identification of genes associated with osteoarthritis by microarray analysis. Mol Med Rep 12: 5211-5216, 2015.

11. Loeser RF, Olex AL, McNulty MA, Carlson CS, Callahan MF, Ferguson CM, Chou J, Leng X and Fetrow JS: Microarray analysis reveals age-related differences in gene expression during the development of osteoarthritis in mice. Arthritis Rheum 64 705-717, 2012

12. Housman G, Havill LM, Quillen EE, Comuzzie AG and Stone AC: Assessment of DNA methylation patterns in the bone and cartilage of a nonhuman primate model of osteoarthritis Cartilage 1: 1947603518759173, 2018.

13. Peffers M, Balaskas P and Smagul A: Osteoarthritis year in review 2017: Genetics and epigenetics. Osteoarthritis Cartilage 26: 304-311, 2018.

14. Zhang Y, Fukui N, Yahata M, Katsuragawa Y, Tashiro T, Ikegawa S and Lee MT: Genome-wide DNA methylation profile implicates potential cartilage regeneration at the late stage of knee osteoarthritis. Osteoarthritis Cartilage 24: 835-843, 2016

15. Rushton MD, Young DA, Loughlin J and Reynard LN Differential DNA methylation and expression of inflammatory and zinc transporter genes defines subgroups of osteoarthritic hip patients. Ann Rheum Dis 74: 1778-1782, 2015.

16. Reynard LN, Bui C, Syddall CM and Loughlin J: CpG methylation regulates allelic expression of GDF5 by modulating binding of SP1 and SP3 repressor proteins to the osteoarthritis susceptibility SNP rs143383. Hum Genet 133: 1059-1073, 2014.

17. Klinger P, Beyer C, Ekici AB, Carl HD, Schett G, Swoboda B, Hennig FF and Gelse K: The transient chondrocyte phenotype in human osteophytic cartilage: A role of pigment epithelium-derived factor? Cartilage 4: 249-255, 2013

18. Aref-Eshghi E, Zhang Y, Liu M, Harper PE, Martin G, Furey A, Green R, Sun G, Rahman P and Zhai G: Genome-wide DNA methylation study of hip and knee cartilage reveals embryonic organ and skeletal system morphogenesis as major pathways involved in osteoarthritis. BMC Musculoskelet Disord 16: 287, 2015.

19. Gautier L, Cope L, Bolstad BM and Irizarry RA: Affy-analysis of affymetrix genechip data at the probe level. Bioinformatics 20 307-315, 2004

20. Diboun I, Wernisch L, Orengo CA and Koltzenburg M: Microarray analysis after RNA amplification can detect pronounced differences in gene expression using limma. BMC Genomics 7: 252, 2006
21. Wang D, Yan L,Hu Q, Sucheston LE, Higgins MJ, Ambrosone CB, Johnson CS, Smiraglia DJ and Liu S: IMA: An R package for high-throughput analysis of Illumina's 450K Infinium methylation data. Bioinformatics 28: 729-730, 2012.

22. Dennis G Jr, Sherman BT, Hosack DA, Yang J, Gao W, Lane HC and Lempicki RA: DAVID: Database for annotation, visualization, and integrated discovery. Genome Biol 4: P3, 2003.

23. Keshava Prasad TS, Goel R, Kandasamy K, Keerthikumar S, Kumar S, Mathivanan S, Telikicherla D, Raju R, Shafreen B, Venugopal A, et al: Human protein reference database-2009 update. Nucleic Acids Res 37: D767-D772, 2009.

24. Livak KJ and Schmittgen TD: Analysis of relative gene expression data using real-time quantitative PCR and the 2 (-Delta Delta C(T)) method. Methods 25: 402-408, 2001

25. Yang F, Zhou S, Wang C, Huang Y, Li H, Wang Y, Zhu Z, Tang J and Yan M: Epigenetic modifications of interleukin-6 in synovial fibroblasts from osteoarthritis patients. Sci Rep 7: 43592, 2017.

26. Zhou X, Chen L, Grad S, Alini M, Pan H, Yang D, Zhen W, Li Z, Huang $S$ and Peng S: The roles and perspectives of microRNAs as biomarkers for intervertebral disc degeneration. J Tissue Eng Regen Med 11: 3481-3487, 2017.

27. Suzuki MM and Bird A: DNA methylation landscapes: Provocative insights from epigenomics. Nat Rev Genet 9: 465-476, 2008

28. Yang $\mathrm{J}$ and Wang $\mathrm{N}$ : Genome-wide expression and methylation profiles reveal candidate genes and biological processes underlying synovial inflammatory tissue of patients with osteoarthritis. Int J Rheum Dis 18: 783-790, 2015.

29. Johnson AA, Akman K, Calimport SR, Wuttke D, Stolzing A and de Magalhaes JP: The role of DNA methylation in aging, rejuvenation, and age-related disease. Rejuvenation Res 15: 483-494, 2012

30. Ezura Y, Sekiya I, Koga H, Muneta T and Noda M: Methylation status of $\mathrm{CpG}$ islands in the promoter regions of signature genes during chondrogenesis of human synovium-derived mesenchymal stem cells. Arthritis Rheum 60: 1416-1426, 2009.

31. Rogers EL, Reynard LN and Loughlin J: The role of inflammation-related genes in osteoarthritis. Osteoarthritis Cartilage 23: 1933-1938, 2015

32. Takahashi A, de Andres MC, Hashimoto K, Itoi E and Oreffo RO Epigenetic regulation of interleukin-8, an inflammatory chemokine, in osteoarthritis. Osteoarthritis Cartilage 23: 1946-1954, 2015.

33. Imagawa K, de Andrés MC, Hashimoto K, Pitt D, Itoi E, Goldring MB, Roach HI and Oreffo RO: The epigenetic effect of glucosamine and a nuclear factor-kappa B (NF- $\mathrm{B}$ ) inhibitor on primary human chondrocytes-implications for osteoarthritis. Biochem Biophys Res Commun 405: 362-367, 2011.

34. Fernández-Tajes J, Soto-Hermida A, Vázquez-Mosquera ME, Cortés-Pereira E, Mosquera A, Fernández-Moreno M, Oreiro N, Fernández-López C, Fernández JL, Rego-Pérez I and Blanco FJ: Genome-wide DNA methylation analysis of articular chondrocytes reveals a cluster of osteoarthritic patients. Ann Rheum Dis 73: 668-677, 2014

35. Xiao JL, Meng JH, Gan YH, Zhou CY and Ma XC: Association of GDF5, SMAD3 and RUNX2 polymorphisms with temporomandibular joint osteoarthritis in female Han Chinese. J Oral Rehabil 42: 529-536, 2015.

36. Ji Q, Xu X, Xu Y, Fan Z, Kang L, Li L, Liang Y, Guo J, Hong T, $\mathrm{Li}$ Z, et al: miR-105/Runx2 axis mediates FGF2-induced ADAMTS expression in osteoarthritis cartilage. J Mol Med (Berl) 94: 681-694, 2016.

37. Leijten JC, Bos SD, Landman EB, Georgi N, Jahr H, Meulenbelt I, Post JN, van Blitterswijk CA and Karperien M: GREM1, FRZB and DKK1 mRNA levels correlate with osteoarthritis and are regulated by osteoarthritis-associated factors. Arthritis Res Ther 15: R126, 2013

38. Han EJ, Yoo SA, Kim GM, Hwang D, Cho CS, You S and Kim WU: GREM1 is a key regulator of synoviocyte hyperplasia and invasiveness. J Rheumatol 43: 474-485, 2016

39. Valverde-Franco G, Lussier B, Hum D, Wu J, Hamadjida A, Dancause N, Fahmi H, Kapoor M, Pelletier JP and Martel-Pelletier J: Cartilage-specific deletion of ephrin-B2 in mice results in early developmental defects and an osteoarthritis-like phenotype during aging in vivo. Arthritis Res Ther 18: 65, 2016.

40. Kaya AI, Lokits AD, Gilbert JA, Iverson TM, Meiler J and Hamm HE: A conserved hydrophobic core in Gail regulates $\mathrm{G}$ protein activation and release from activated receptor. J Biol Chem 291: 19674-19686, 2016 
41. Diehl SA, McElvany B, Noubade R, Seeberger N, Harding B, Spach $K$ and Teuscher C: G proteins galphail/3 are critical targets for bordetella pertussis toxin-induced vasoactive amine sensitization. Infect Immun 82: 773-782, 2014.

42. Rivetti S, Lauriola M, Voltattorni M, Bianchini M, Martini D, Ceccarelli C, Palmieri A, Mattei G, Franchi M, Ugolini G, et al: Gene expression profile of human colon cancer cells treated with cross-reacting material 197, a diphtheria toxin non-toxic mutant. Int J Immunopathol Pharmacol 24: 639-649, 2011.

43. Mabey T, Honsawek S, Saetan N, Poovorawan Y, Tanavalee A and Yuktanandana P: Angiogenic cytokine expression profiles in plasma and synovial fluid of primary knee osteoarthritis. Int Orthop 38: 1885-1892, 2014

44. Gao W, Sweeney C, Walsh C, Rooney P, McCormick J, Veale DJ and Fearon U: Notch signalling pathways mediate synovial angiogenesis in response to vascular endothelial growth factor and angiopoietin 2. Ann Rheum Dis 72: 1080-1088, 2013.

45. Yi J, Jin Q, Zhang B, Wu X and Ge D: Gremlin-1 concentrations are correlated with the severity of knee osteoarthritis. Med Sci Monit 22: 4062-4065, 2016.
46. Fan Y, Chen J, Yang Y, Lin J and Wu Z: Genome-wide expression and methylation profiling reveal candidate genes in osteoarthritis. Clin Exp Rheumatol 35: 983-990, 2017.

47. Hopwood B, Tsykin A, Findlay DM and Fazzalari NL: Microarray gene expression profiling of osteoarthritic bone suggests altered bone remodelling, WNT and transforming growth factor- $\beta$ /bone morphogenic protein signalling. Arthritis Res Ther 9: R100, 2007.

48. Liao L, Zhang S, Gu J, Takarada T, Yoneda Y, Huang J, Zhao L, Oh CD, Li J, Wang B, et al: Deletion of Runx2 in articular chondrocytes decelerates the progression of DMM-induced osteoarthritis in adult mice. Sci Rep 7: 2371, 2017.

This work is licensed under a Creative Commons Attribution-NonCommercial-NoDerivatives 4.0 International (CC BY-NC-ND 4.0) License. 